\title{
Greater Physician Involvement Improves Coding Outcomes in Endobronchial Ultrasound-Guided Transbronchial Needle Aspiration Procedures
}

\author{
Anilkumar Pillai Andrew R.L. Medford \\ North Bristol Lung Centre, Southmead Hospital, Bristol, UK
}

\section{Key Words}

Endobronchial ultrasound-guided transbronchial needle aspiration - Coding . Payment by results · Tariff

\begin{abstract}
Background: Correct coding is essential for accurate reimbursement for clinical activity. Published data confirm that significant aberrations in coding occur, leading to considerable financial inaccuracies especially in interventional procedures such as endobronchial ultrasound-guided transbronchial needle aspiration (EBUS-TBNA). Previous data reported a $15 \%$ coding error for EBUS-TBNA in a UK service. Objectives: We hypothesised that greater physician involvement with coders would reduce EBUS-TBNA coding errors and financial disparity. Methods: The study was done as a prospective cohort study in the tertiary EBUS-TBNA service in Bristol. 165 consecutive patients between October 2009 and March 2012 underwent EBUS-TBNA for evaluation of unexplained mediastinal adenopathy on computed tomography. The chief coder was prospectively electronically informed of all procedures and cross-checked on a prospective database and by Trust Informatics. Cost and coding analysis was performed using the 2010-2011 tariffs. Results: All 165 procedures (100\%) were coded correctly as verified by Trust Informatics. This compares favourably with the $14.4 \%$ coding inaccuracy rate for EBUS-TBNA in a previous UK prospective cohort study [odds ratio $201.1(1.1-357.5), p=0.006]$. Projected income loss was GBP 40,000 per year in the previous
\end{abstract}

study, compared to a GBP 492,195 income here with no coding-attributable loss in revenue. Conclusions: Greater physician engagement with coders prevents coding errors and financial losses which can be significant especially in interventional specialties. The intervention can be as cheap, quick and simple as a prospective email to the coding team with cross-checks by Trust Informatics and against a procedural database. We suggest that all specialties should engage more with their coders using such a simple intervention to prevent revenue losses.

Copyright $\odot 2013$ S. Karger AG, Base

\section{Introduction}

Medical records are an important aspect of patient care. Not only do they convey the clinical information associated with the patient but they can also be used to report and monitor the activity of hospital services. The Health Informatics Unit of the Royal College of Physicians, London has developed generic medical record keeping standards for physicians that can be applied to all patients in hospital practice. The purpose of these standards is to maximise patient safety and quality of care, support best professional practice and to assist compliance with Information Governance and NHS Litigation Authority Standards. They define good practice for medical records and address the broad requirements that apply to all clinical note keeping [1].

\section{KARGER}

E-Mail karger@karger.com

www.karger.com/res (c) 2013 S. Karger AG, Basel

0025-7931/13/0855-0417\$38.00/0
Dr. Andrew R.L. Medford

North Bristol Lung Centre, Southmead Hospital

Westbury-on-Trym, Bristol BS10 5NB (UK)

E-Mail andrewmedford@ hotmail.com 
Medical records form an integral part of coding of hospital activity and the accuracy of coding reflects much on the adequacy of entries in the medical notes. Under payment by results $(\mathrm{PbR})$, hospital trusts have a financial incentive to ensure accurate coding of the services they have provided to the patient. $\mathrm{PbR}$ ostensibly aims to pay hospitals for the work they do and reward them for their efficiency and quality. Aberrations in coding are well known and have been described by the Audit Commission [2] They report that coding errors continue to plague the Healthcare Resource Group (HRG) system, thus affecting accuracy of payment. The average HRG error report rate was $8.1 \%$ with a range of $1-40 \%$ with considerable local variation. However, in most cases the net financial impact was close to zero, indicating that losses suffered by hospital trusts were balanced by a gain to the local Primary Care Trust. The level of coding errors is worrying given the financial pressures faced by all hospital trusts.

Most of the factors that affect the coding accuracy reflect the need for greater clinician engagement to improve the standard of records and other source documentation, validate codes and give direction on identifying and coding comorbidities. Some of the other factors which should be highlighted include the continued need to improve training and development programmes for clinical coders and to develop local audit and assurance programmes. The 2010/2011 Audit Commission report once again emphasised the need for better senior leadership in the organisation and greater clinical involvement in this process [3].

In North America a system of diagnosis-related groups (DRGs) has been utilised for prospective payment systems and reimbursement by Medicare since the mid1980s, but there have been reports of Medicare reimbursement inaccuracy due to DRG errors, occurring at a rate of over 20\% [4]. They also described 'DRG creep' where over $60 \%$ of the errors led to the over-reimbursement of hospitals covered by Medicare and evidence of systematic biases affecting some specialities at patient and facility level $[5,6]$. This is somewhat similar to the NHS experience cited in the Audit Commission report.

The potential for coding inaccuracies in interventional specialities has also been documented in the report, and this has greater financial effects, typically because the tariffs are higher. The interventional pulmonological procedure endobronchial ultrasound-guided transbronchial needle aspiration (EBUS-TBNA) tariff is about 7 times the conventional bronchoscopy tariff and, therefore, provides a good model to illustrate the effects of coding inaccuracies. In an earlier UK EBUS-TBNA coding study, more than $15 \%$ of the EBUS-TBNA HRG codes were inaccurate [7]. If EBUS-TBNA is coded as a conventional bronchoscopy, it costs the Trusts GBP 6,144 per year although it is cost saving to the NHS (GBP 107,824 per year) and the Primary Care Trust (GBP 113,968 per year). Similar coding errors have been described in the UK in local anaesthetic thoracoscopy, another interventional pulmonological procedure, with a coding error rate of nearly $70 \%$ and financial disparity of nearly GBP 70,000 [8].

In this prospective quality improvement study we set out to analyse whether greater physician involvement in the coding process improved coding outcomes with regard to EBUS-TBNA specifically. Our hypothesis was that greater physician involvement using a simple prospective coding cross-checking system would improve coding outcomes for EBUS-TBNA and reduce financial disparity.

\section{Methods}

Over a 16-month period from November 2010 to February 2012, 165 consecutive patients underwent day-case EBUS-TBNA at a UK teaching hospital for the investigation of unexplained mediastinal lymphadenopathy on computed tomography and/or positron emission tomography. EBUS-TBNA was performed as described previously [7]. Institutional Review Board approval was obtained for the study.

As standard practice the EBUS-TBNA procedure notes are handwritten on an appropriately coloured paper report and filed in the medical records. The Trust coders refer to the handwritten medical notes on coloured bronchoscopy report sheets for evidence of the EBUS-TBNA procedure and that biopsies have been taken.

For the intervention, anonymised patient and procedure details (EBUS-TBNA as the procedure with the anonymised hospital number, the assigned code was not sent) were emailed securely to the Trust Coding lead after every procedure list by the operator (A.R.L.M.). Every month, the Trust Informatics lead would email the final coding outcomes and tariffs for all the EBUS-TBNA patients. These were cross-checked against a prospective anonymised procedure database. The 'predicted' HRG codes were derived from the National Tariff 2011-2012 [9], and for EBUS-TBNA, a tariff of GBP 2,983 per procedure was applied. 'Predicted' (as expected from the National Tariff) and 'actual' (as coded on the system by the Trust coders) costings according to the PbR tariff were derived from the selected HRG codes. The time of finishing the list and the approximate time of completing the coder email and maintenance of the cross-checking procedural database were recorded.

Data were compared to a previous EBUS-TBNA coding study [7] as a non-intervention coding control study. Statistical analysis was performed with GraphPad Prism (version 5) software, using contingency table analysis and Fisher's exact test with data expressed as odds ratio with 95\% confidence interval expressed in parentheses. $\mathrm{p}<0.05$ was deemed significant. Differences in finan- 
cial loss were calculated using a tariff of GBP 503 for conventional bronchoscopy and GBP 2,983 for EBUS-TBNA [9]. The primary outcome was coding accuracy. The estimated gross financial error was calculated by deriving the mean error per episode and multiplying the effect.

\section{Results}

One hundred and sixty-five patients were referred for EBUS-TBNA, of which $24 \%$ were tertiary referrals. All patients were successfully treated with a day-case procedure yielding EBUS-TBNA biopsies with no requirement for overnight admission and no complications. Results were compatible with the expected performance [10] with an overall sensitivity of over $90 \%$, a negative predictive value of $89 \%$, and an accuracy of $95 \%$ for mediastinal lymph node metastases (prevalence of 56\%).

All 165 patients were coded correctly after the intervention described with $100 \%$ coding accuracy. In the previous UK study of 52 patients [7], 8 (15.4\%) were coded incorrectly, which was significant (odds ratio 201.1; $1.1-$ $357.5 ; \mathrm{p}=0.006$ ), with a projected EBUS-TBNA-attributable income of GBP 183,816; the financial loss to the NHS Trust was GBP 23,200 (GBP 40,000 per year). In this study, there was no financial loss to the Trust as all coding episodes were accurate. The projected EBUS-TBNA-attributable revenue to the Trust was GBP 492,195.

\section{Discussion}

In this quality improvement study, we have also demonstrated that greater physician involvement in the coding process with validation of the codes applied significantly improves the coding outcome in EBUS-TBNA procedures. We have shown that with greater clinician involvement in the coding process the coding errors are minimised, and consequently there is no financial loss. HRG coding allocation errors occur frequently. The potential financial loss due to coding errors has significant implications for service providers and commissioners. Our earlier data described discrepancies in HRG coding for interventional pulmonological procedures in the UK of between 15 and $70 \%$ of cases $[7,8]$. These occurrences are described in other interventional medical and surgical specialties. In otolaryngology, error rates of nearly $25 \%$ have been noted, equating to an average revenue loss of GBP 174 per patient due to frequent miscoding of highly complex procedures [11]. The net effect of these coding

Greater Physician Involvement Improves

Coding Outcomes inaccuracies on $\mathrm{PbR}$ funding per patient episode appeared to be small; however, the magnitude of the financial inaccuracies cannot be overlooked. This also highlighted the difficulties with the HRG coding system which has not kept pace with the changes in health care particularly in interventional specialities, where the magnitude of financial loss is much more.

$\mathrm{PbR}$ aims to improve efficiency in the NHS. Accurate coding is essential to ensure that Trusts are paid properly for the work they do [12]. Despite the more recent widespread use of EBUS-TBNA compared to 10 years ago, it cannot be assumed that EBUS-TBNA coding would have improved in the NHS given the accumulation of evidence to the contrary in other procedures. Moreover, correct coding has implications for all rationed health care systems, not just the NHS and therefore is of international importance. Staff involved in coding should be welltrained and must effectively interact with clinicians. Good communication between coding department and clinicians is a must for the system to be successful. The complexity of the system has been demonstrated in an article by Jameson and Reed [13] with regard to orthopaedic procedures.

Accurate coding is the key to PbR- and HRG-related tariff reimbursement in the health sector especially for interventional procedures such as EBUS-TBNA but also conventional TBNA which is more commonplace [14, 15]. However, coding inaccuracies are not new. Previous studies have reported inaccuracy rates of about $9 \%$ with the DRG coding system in the USA and about $20 \%$ with the HRG system in the UK [4-6]. The Audit Commission have found coding variation across Trusts in England of between 0.3 and 52\%. In the 2007/2008 report the net financial loss was minimal, but the gross financial error was about $4.7 \%$ of the total budget of GBP 72.8 million, thus demonstrating a minimal net but significant gross financial error [2]. The most common reasons for coding errors were identified as poor quality documentation in the medical notes, electronic patient records and discharge summaries and coder training issues. Interestingly one of the reasons given was the lack of an adequate level of clinician involvement and validation in the whole process.

Other coding studies have looked at clinician engagement in different ways to improve coding accuracy. A general medical and rheumatology quality improvement study demonstrated that physician interaction with the coders helped to achieve 95\% coding accuracy of data [16]. Another quality improvement study in otolaryngology achieved a similar 95\% coding accuracy by greater engagement through weekly meetings between clinicians 
and coders to discuss difficult cases [17]. The interventions in our study did not incur extra cost and could be achieved with minimal effort (compared to some of the other interventions reported previously such as weekly meetings between coders and clinicians). In the current resource-strapped economic climate, financial prudence is necessary and accurate costing for procedures is essential. The potential impact of inaccurate coding has an enormous impact on directorate revenue, budgets, service planning and development, commissioning and clinical audit. Our study reports a very simple, quick but effective way to minimise coding errors on interventional or procedural lists, which could be applied to other interventional/surgical specialities.

We acknowledge that one of the limitations of this intervention could be the time devoted to it by the clinician. In a busy clinical set-up with consultants leading from the front at multiple levels, time is a scarce commodity. Devoting extra time to cross-checking codes which had been applied could potentially impact on consultant time. This also depends on the volume of procedures. However, maintaining an accurate anonymised procedural database for audit purposes is essential for audit and revalidation. A brief electronic communication to the coder does not take very long and could be delegated to another member of the clinical team if needed. We, therefore, do not believe this intervention should be impractical or time consuming if designed appropriately and that such results can be reproduced in a large centre. Cross-checking to confirm if the correct codes have been applied by Trust Informatics is an important safeguard. Cross-checking against own procedural databases could be more time-consuming for clinicians depending on volume, but with shared folder access to secure areas in hospital IT systems, this could be delegated to a variety of clinical team members to make it less onerous. It is acknowledged that this might lead to an increase in costs of consultant/appointed deputy time, but this would be more than offset by the cost savings for correct coding of all procedures, which would be multiplicative. We focussed on EBUS-TBNA in this study yielding a GBP 40,000 saving for this procedure in particular. During the study, estimated extra time incurred (to email the coder, check the return and enter and maintain the procedural database for cross-checking) by the clinician was less than $1 \mathrm{~h}$ per week equating to less than 0.25 programmed activities per week (out of a 10 programmed activities week) or GBP 2,125 per year assuming an average consultant salary of GBP 85,000 (0.25/10 $\times 85,000)$. A GBP 40,000 yield more than offsets the GBP
2,125 per year estimated cost attributable to administration. We acknowledge that this study does not specifically address the important issues of conservatively managed emergency work and also accurate coding of morbidities, both of which are fundamental to accurate coding too and require further study. We also acknowledge that a historical control data set has been used for comparison in this study. It should be noted that the coder in this study had equivalent experience but was not the same as the previous coder. We accept, therefore, that it is not possible to completely exclude slightly different levels of coder experience as confounding factors, but we have attempted to minimise this. However, because the coder was not the same, effects of feedback on the coder in the previous study and accrued experience between the studies could not have been confounding factors. Some clinicians utilise electronic billing methods, and this intervention will have less impact on such systems where the coding assignation function of coders has been replaced by physicians. Finally, in terms of the challenge of using this system for the same procedure by multiple sites and operators in the same centre, this can be reproduced. Trust Informatics monitors activity in line with expected activity for the procedure and can flag up any anomalies with the operator(s) and finance department. In addition, although the operator or deputy is required to email the details, the central coder emails the operator in the event of no email being sent as a further crosscheck.

The National Tariff generally determines the remuneration for the procedural activity. If the same procedure is undertaken in theatre with a general anaesthetic (EBUS-TBNA for example), the same tariff will apply; however, sometimes a slightly increased local tariff can be adopted if the commissioner for the service can be satisfied for this need. Regarding other procedures that can only be done in theatre under general anaesthesia, this increased cost and complexity should be reflected in a higher tariff, but this is not always the case.

Other strategies to minimise coding inaccuracies are needed at different levels. Improving documentation and appropriate coder training are obviously important. However, it is doubtful if these measures alone could cause as significant an impact on coding accuracy and are not a replacement for the need for some sort of independent and Trust cross-checking system as internal quality controls. Coding systems need to be reviewed and updated in line with the rapid progress made in interventional specialities [18], and they also need to be simplified to minimise code variations for the same procedure. 
In summary, we have demonstrated that a simple intervention can achieve greater physician engagement with coders and improve coding outcomes in a UK respiratory centre providing a tertiary EBUS-TBNA service. This is of particular importance in interventional specialities where the potential for financial loss is of a higher magnitude. A simple prospective cross-checking system can achieve better outcomes with no extra cost and minimal effort. This intervention should be replicated in other specialities and in other centres and should be evalu- ated in a national quality improvement study with large services with multiple operators to confirm its reproducibility.

\section{Acknowledgements}

We would like to thank the Trust Coding and Informatics Teams at the North Bristol NHS Trust for their contribution.

\section{References}

1 Royal College of Physicians of London Health Informatics Unit. A Clinician's Guide to Record Standards. 2. Standards for the Structure and Content of Medical Communications when Patients Are Admitted to Hospital. London, NHS Digital and Health Information Policy Directorate, 2008. http://www. rcoa.ac.uk/docs/Clinicians-Guide-Part-2Standards.pdf.

2 Audit Commission. PbR Data Assurance Framework 2007/08: findings from the first year of the national clinical coding audit programme. http://www.audit-commission.gov. uk/Products/NATIONAL-REPORT/ CD8608E5-A7D9-4a5a-B0F3-C161B76 DE630/PbRreport.pdf. 2007/8.

3 Audit Commission. Improving coding, costing and commissioning: annual report on the $\mathrm{PbR}$ data assurance programme 2010/11. http://www.audit-commission.gov.uk/ health/paymentbyresults/reportsandstudies/ pbr2011/Pages/pbr2011_copy.aspx.

4 Hsia DC, Krushat WM, Fagan AB, Tebbutt JA, Kusserow RP: Accuracy of diagnostic coding for Medicare patients under the prospective-payment system. N Engl J Med 1988;318: 352-355.

5 Hsia DC: Accuracy of Medicare reimbursement for cardiac arrest. JAMA 1990;264:5962.
6 Muldoon JH: Structure and performance of different DRG classification systems for neonatal medicine. Pediatrics 1999;103(1 suppl E):302-318

7 Medford AR, Agrawal S, Free CM, Bennett JA: A performance and theoretical cost analysis of endobronchial ultrasound-guided transbronchial needle aspiration in a UK tertiary respiratory centre. QJM 2009;102:859864.

8 Medford AR, Agrawal S, Free CM, Bennett JA: Retrospective analysis of Healthcare Resource Group coding allocation for local anaesthetic video-assisted 'medical' thoracoscopy in a UK tertiary respiratory centre. QJM 2009;102:329-333.

9 Department of Health. Confirmation of payment by results (PbR) arrangements for 2011-12. http://www.dh.gov.uk/en/Publicationsandstatistics/Publications/PublicationsPolicyAndGuidance/DH_124356. 2011.

10 Detterbeck FC, Jantz MA, Wallace M, Vansteenkiste J, Silvestri GA: Invasive mediastinal staging of lung cancer: ACCP evidence-based clinical practice guidelines (2nd edition). Chest 2007;132(3 suppl):202S-220S.

-11 NouraeiSA, O'Hanlon S, Butler CR, Hadovsky A, Donald E, Benjamin E, et al: A multidisciplinary audit of clinical coding accuracy in otolaryngology: financial, managerial and clinical governance considerations under payment-by-results. Clin Otolaryngol 2009; 34:43-51.
12 Newbold D: Caring about the costs. Nurs Stand 2006;20:24-25.

13 Jameson S, Reed MR: Payment by results and coding practice in the National Health Service. The importance for orthopaedic surgeons. J Bone Joint Surg Br 2007;89:14271430.

14 Medford AR, Agrawal S, Free CM, Bennett JA: A prospective study of conventional transbronchial needle aspiration: performance and cost utility. Respiration 2010;79: 482-489.

15 Trisolini R, Patelli M, Gasparini S: While waiting to buy a Ferrari, do not leave your current car in the garage! Respiration 2010;79: 452-453.

-16 Nallasivan S, Gillott T, Kamath S, Blow L, Goddard V: Physician involvement enhances coding accuracy to ensure national standards: an initiative to improve awareness among new junior trainees. J R Coll Physicians Edinb 2011;41:106-108.

17 Carr ER, Pillai A: Re: A multidisciplinary audit of clinical coding accuracy in otolaryngology: financial, managerial and clinical governance considerations under payment-by-results. Clin Otolaryngol 2009;34:259-260.

18 Medford AR: Endobronchial ultrasoundtransbronchial needle aspiration and lung cancer. J R Coll Physicians Edinb 2009;39:91. 